\title{
Development of $\mathrm{NO}_{2}$ Gas Sensor Using Colorimetric Film and Organic Solar Cell in Self-powered System
}

\author{
Kyungnam Kang ${ }^{1}$, Kwangmin $\mathrm{Na}^{2}$, Jung-Yong Lee ${ }^{2}$, Inkyu Parkr ${ }^{\text {* }}$ \\ 1 Department of Mechanical Engineering, Korea Advanced Institute of Science and Technology \\ (KAIST), 291 Daehak-ro, Yuseong-gu, Daejeon 34141, Republic of Korea \\ 2 Graduate school of EEWS, Korea Advanced Institute of Science and Technology (KAIST), 291 \\ Daehak-ro, Yuseong-gu, Daejeon 34141, Republic of Korea \\ inkyu@kaist.ac.kr
}

\begin{abstract}
This paper demonstrates the fabrication of a self-powered $\mathrm{NO}_{2}$ gas sensor using a visible light source. This sensor is composed of a N,N,N',N'-tetramethyl-p-phenylenediamine (TMPD) colorimetric film on top of an organic photovoltaic cell. The TMPD colorimetric material changes its color when it is exposed to $\mathrm{NO}_{2}$. Based on this transmittance change, the output current from the solar cell beneath the film is used to build a gas detection system. Conventional colorimetric gas sensors use a singlewavelength light source and a detector. However, the sensor system in this report uses a visible light source (to emulate ambient light), thereby avoiding the use of additional power sources for the sensor. To increase the sensitivity of the device, a micro post array (with $20 \mu \mathrm{m}$ in diameter) was formed on the sensing film. The larger the aspect ratio of the micro post, the better the performance of the sensor. Further enhancement of the performance was observed by incorporating nanowires on the micro post. $\mathrm{NO}_{2}$ gas was successfully detected at concentrations of $1 \sim 20 \mathrm{ppm}$ with this system. By modularizing this sensor, users can easily detect gases that threaten their personal health without the inconvenience of replacing a battery.
\end{abstract}

Key words: self-powered, gas sensor, nitrogen dioxide, organic solar cell, colorimetric material

\section{Introduction}

Gas leakages in the workplace or in factories threaten personal health. Therefore, the detection of toxic gases surrounding people is an important issue. Many types of equipment (based on optical methods, mass spectrometry, resistance changes and electrochemical reaction) are used to measure toxic gases, but only resistive type gas sensors and electrochemical gas sensors are becoming popular as portable sensors due to their ability to be miniaturized [1]. Recently, a colorimetric sensor was developed to solve the power consumption and the fabrication process issues typically associated to portable gas sensors [2]. However, these colorimetric sensors, in which the color of a material changes in the presence of a gas, use a detector and a specific singlewavelength light source [3]. Therefore, these sensors still consume relatively large amount of power and the size of them is relatively big.

This study demonstrates the construction of a self-powered $\mathrm{NO}_{2}$ sensor composed of a transparent film and an organic solar cell. The colorimetric material reacts with the toxic gas to change its transmittance in the visible region, thereby causing a change in the output of the solar cell. This approach allows the detection of $\mathrm{NO}_{2}$ without any additional power consumption, i.e., only with the use of ambient white light. In addition, the performance of the sensor could be improved by forming micro/nano structures on the surface of the sensing film, and a practical application of this self-powered gas sensing system is demonstrated.

\section{Fabrication of Sensing Film and Solar Cell}

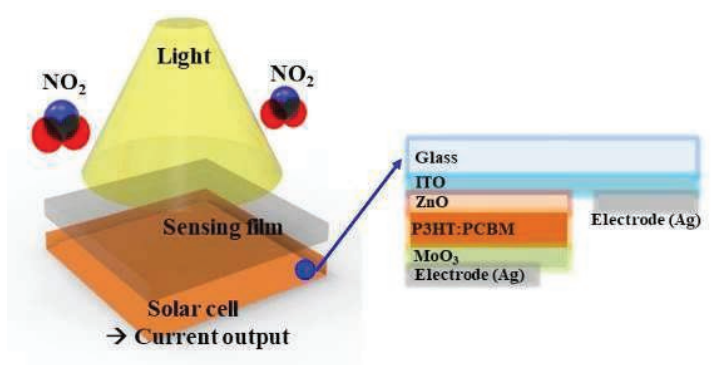

Fig 1. Schematic representation of the self-powered gas sensor and the organic solar cell.

The components of the sensor system are shown in Fig 1. There is a transparent film on top of the organic solar cell; this film serves as the colorimetric material that responds to the target toxic gas. Organic solar cells were fabricated by spin coating for P3HT (photoactive layer), and thermally evaporating for Ag (electrode). For the sensing film, a micro post array film of polydimethylsiloxane (PDMS) was made by molding method using SU-8 template. Subsequently, a mixed solution of $\mathrm{N}, \mathrm{N}, \mathrm{N}$ ',N'-tetramethyl-p-phenylenediamine 
(TMPD) and Isopropyl alcohol was coated onto PDMS film by spray coating. For a more enhanced surface area, the $\mathrm{ZnO}$ nanowires were synthesized on the micro post through hydrothermal growth before spray coating.

\section{Measurement of Sensing Film Transmittance}

The transmittance of TMPD-coated and uncoated films were checked by a UV-vis spectrometer before and after the injection of $\mathrm{NO}_{2}$. Twenty $\mathrm{ppm}$ of $\mathrm{NO}_{2}$ were injected for 30 minutes, and then the transmittance of the film was measured.

\section{Gas Experiment Procedure}

During the gas test, the sensor system was put in a chamber and light was allowed to enter. The $\mathrm{NO}_{2}$ gas and the synthesized air were flowed into the chamber by a Mass Flow Controller (MFC) while irradiating light with an intensity of $235 \mathrm{~W} / \mathrm{m}^{2}$ through a solar illuminator The current change of the solar cell was measured in real time through a source meter with no external excitation voltage applied to the sensor.

\section{Transmittance Results}
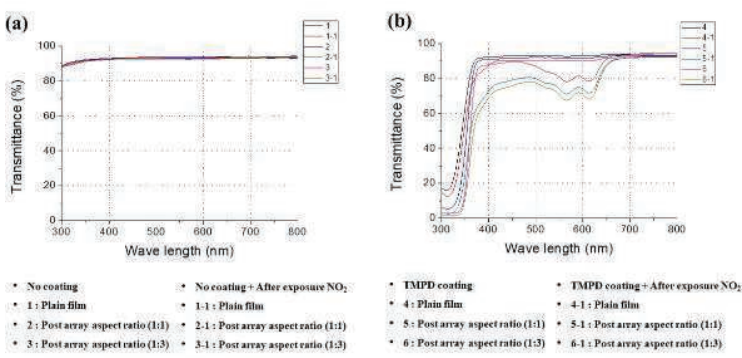

Fig 2. Transmittance of the film after exposure to 20 ppm of $\mathrm{NO}_{2}$, (a) no coated film, (b) TMPD-coated film.

In the absence of TMPD, there is no change in the transmittance even when the sensor is exposed to $\mathrm{NO}_{2}$. However, in the film with TMPD, the transmittance of the irradiated light decreased in the light wavelength range of 500$600 \mathrm{~nm}$ after gas exposure, and the color was visually changed.

\section{Gas Sensing Results and Application}

The transmittance of the TMPD material, in the visible light region, changed when it reacted with $\mathrm{NO}_{2}$. As shown in Fig 3 (a), the sensitivity increased when the micro post array was formed. Furthermore, the larger the aspect ratio of the micro post, the higher the sensitivity that was achieved. This is presumably because the effective surface area of the colorimetric material causing the change in transmittance was increased. Furthermore, the performance of the sensor was also improved by the incorporation of nanowires to the micro post array. This is because the surface effect was maximized with the incorporation of nanowires. The sensitivity increased with increasing nanowire lengths. The different lengths of these nanowires grown on the surface of the micro post were achieved by varying the growth time of the nanowires.

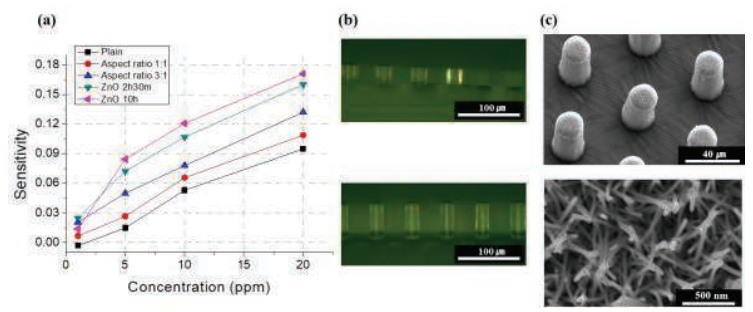

Fig 3. (a) Sensitivity ( $\Delta I / I 0, \Delta I$ : current change of solar cell, $I_{0}$ : the current value before gas exposure) to $\mathrm{NO}_{2}$, (b) optical image of the PDMS micro post array (aspect ratio 1:1 (up), 3:1(down)), (c) SEM image of the post array with the grown $\mathrm{ZnO}$ nanowires (growth time : $2 \mathrm{~h} 30 \mathrm{~min}$ ).

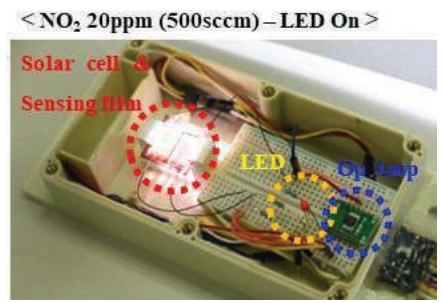

Fig 4. The self-powered gas alarm system for $\mathrm{NO}_{2} 20$ ppm using an Op Amp and a light source.

We constructed a sensor system for a practical application and demonstrated its use. An Op Amp was used for signal amplification, and the source used to drive this system was a visible light source. An LED was successfully tested to light up when a threshold concentration of $20 \mathrm{ppm}$ of $\mathrm{NO}_{2}$ was injected into the chamber as shown in Fig 4.

\section{Conclusions}

In this paper, a self-powered $\mathrm{NO}_{2}$ gas sensor was developed. The color of a TMPD film changed when in the presence of $\mathrm{NO}_{2}$; this change subsequently resulted a useable solar cell output signal. Therefore, $\mathrm{NO}_{2}$ (in concentrations of $1,5,10,20$ ppm) could be detected with the use of a visible light source without any power consumption. Furthermore, the performance of the gas sensor was improved by forming micro/nano structures. Finally, a simple gas alarm system was demonstrated with the use of an Op Amp.

\section{References}

[1] I. Lee, S-J. Choi, K-M. Park, S. Lee, S. Choi, I-D. Kim, C. Park, The stability, sensitivity and response transients of $\mathrm{ZnO}, \mathrm{SnO} 2$, and $\mathrm{WO} 3$ sensors under acetone, toluene and $\mathrm{H} 2 \mathrm{~S}$ environments, Sens. Actuator B-Chem., 197, 300 (2014); doi.org/10.1016/j.snb.2014.02.043

[2] T. Mayr, T. Abel, B. Enko, S. Borisov, C. Konrad, S. Kostler, B. Lamprecht, S. Sax, E. List, I. Klimant, A planar waveguide optical sensor employing simple light coupling, Analyst, 134, 1544 (2009); doi: 10.1039/B904536H

[3] J. Courbat, D. Briand, J. Wollenstein, N. de Rooij, Polymeric foil optical waveguide with inkjet printed gas sensitive film for colorimetric sensing, Sens. Actuator B-Chem., 160, 910 (2011); doi.org/10.1016/j.snb.2011.09.001 